\title{
EIGENVALUE MULTIPLICITIES OF PRODUCTS OF COMPANION MATRICES*
}

\author{
ERIC S. $\mathrm{KEY}^{\dagger}$ AND HANS VOLKMER ${ }^{\dagger}$
}

\begin{abstract}
It is shown that under suitable conditions an eigenvalue of a product of companion matrices has geometric multiplicity equal to one. The result is used to show that for a class of Random Walks in Periodic Environments recurrence is equivalent to a product of companion matrices having 1 as an eigenvalue of algebraic multiplicity greater than one.
\end{abstract}

Key words. Matrix products, Eigenvalue multiplicities, Companion matrices, Random walk in a periodic environment.

AMS subject classifications. 15A18, 15A21, 60J10.

1. Introduction. Consider a companion matrix of the form

$$
B=\left[\begin{array}{cccccc}
a_{1} & a_{2} & a_{3} & \ldots & a_{n-1} & a_{n} \\
1 & 0 & 0 & \ldots & 0 & 0 \\
0 & 1 & 0 & \ldots & 0 & 0 \\
\ldots & \ldots & \ldots & \ldots & \ldots & \ldots \\
0 & 0 & 0 & \ldots & 1 & 0
\end{array}\right]
$$

If $x$ is an eigenvalue of $B$, then it is well-known that all corresponding eigenvectors are multiples of $\left[x^{n-1}, x^{n-2}, \ldots, x, 1\right]^{t}$. In particular, each eigenvalue of a companion matrix is geometrically simple, that is, its geometric multiplicity is equal to one. We also see that if $B_{1}, B_{2}, \ldots, B_{k}$ are companion matrices of the same dimension, then they have an eigenvalue in common if and only if they have an eigenvector in common. If $x$ is the common eigenvalue, then $x^{k}$ is an eigenvalue of the product $B_{1} B_{2} \ldots B_{k}$. However, in contrast to the situation of a single companion matrix, the eigenvalue $x^{k}$ of $B_{1} B_{2} \ldots B_{k}$ may have geometric multiplicity greater than one. In this paper we shall give conditions on the matrices $B_{j}$ that ensure that $x^{k}$ is geometrically simple. We also present a method to calculate the algebraic multiplicity of $x^{k}$ without having to multiply out the matrices $B_{1}, B_{2}, \ldots, B_{k}$. These results are contained in Sections 3 and 4 of this paper.

The problem of determining the multiplicities of an eigenvalue of a product of companion matrices appears in the study of Random Walks in a Periodic Environment (RWPE), a type of Markov Chain studied in [5] in discrete time and in [1] in continuous time. A RWPE is described by a finite number of companion matrices, and the knowledge of the multiplicities of an eigenvalue of their product is necessary in order to distinguish between its recurrent or transient behavior. For the convenience of the reader we present a short introduction to the parts of the theory of RWPE's that are

\footnotetext{
*Received by the editors 9 June 2003. Accepted for publication 1 April 2004. Handling Editor: Stephen J. Kirkland.

${ }^{\dagger}$ Department of Mathematical Sciences, University of Wisconsin-Milwaukee, PO Box 413, Milwaukee, Wisconsin 53201-0413, USA (ericskey@uwm.edu, volkmer@uwm.edu).
} 
relevant to us in Section 2. In Section 5 we apply our general results to such Random Walks.

Products of companion matrices also occur in other areas of mathematics. Consider, for example, the $n^{\text {th }}$ order recursion for the scalar sequence $\left(x_{m}\right)$,

$$
x_{m}=a_{m, 1} x_{m-1}+\cdots+a_{m, n} x_{m-n}+f_{m} .
$$

This recursion is equivalent to a first order recursion for a $n$-dimensional vector sequence $\left(X_{m}\right)$,

$$
X_{m}=B_{m} X_{m-1}+F_{m}
$$

Here $B_{m}$ is an $n \times n$ companion matrix whose first row is

$$
\left[a_{m, 1}, \ldots, a_{m, n}\right]
$$

Thus solutions of the recursion can be expressed in terms of products of companion matrices, and the rates of growth of these solutions are determined in part by the structure of the eigenspaces of these products; see [6, pp. 63-85]. The case where the coefficients of (1.2) are periodic in $m$ has been discussed by several authors, including [2, pp. 178-213], [6, pp. 63-85] and [7, pp. 335-350]. Such difference equations naturally give rise to finite products of companion matrices, yet little seems to be known about the eigenspaces of such products, even in the case where these companion matrices have an eigenvalue in common. For example, there seems to be no mention of the behavior of such matrix products in [4].

2. Random Walks in a Periodic Environment. A Markov chain $X$ on the integers is called a Random Walk if its transition matrix $P(x, y):=\operatorname{Pr}\left(X_{n+1}=\right.$ $\left.y \mid X_{n}=x\right)$ satisfies the condition

$$
P(x, y)=P(x+1, y+1)
$$

for all pairs of integers $(x, y)$. If we interpret $X_{n}$ as the position of a particle on the integer lattice, this condition on $P(\cdot, \cdot)$ means that the statistical mechanism determining how $X_{n}$ changes does not depend on the current location. It is wellknown that if we define

$$
\mu=\sum_{a} a P(x, x+a)
$$

then we have the following theorem.

THEOREM 2.1. Let $X$ be a random walk.

- If $\mu>0$ then $\operatorname{Pr}\left(\lim _{t \rightarrow \infty} X_{t}=+\infty\right)=1$ and we say that the random walk is transient.

- If $\mu=0$ then $\operatorname{Pr}\left(-\infty=\liminf _{t \rightarrow \infty} X_{t}<\limsup _{t \rightarrow \infty} X_{t}=+\infty\right)=1$ and we say that the random walk is recurrent.

- If $\mu<0$ then $\operatorname{Pr}\left(\lim _{t \rightarrow \infty} X_{t}=-\infty\right)=1$ and we say that the random walk is transient. 
If we want the statistical mechanism to vary periodically with the current location $x$, then we require instead that

$$
P(x, y)=P(x+N, y+N)
$$

for some positive integer $N$ and all pairs of integers $(x, y)$. The least such $N$ is called the period of the Markov chain $X$. In this case the Markov chain is called a Random Walk in a Periodic Environment (RWPE) with period $N$.

The recurrence and transience behavior of RPWE's in general is discussed in $[5$, pp. 552-555]. There we find that the author defines $e(x ; a):=P(x, x+a)$ and imposes the following three conditions on $e(\cdot, \cdot)$ :

There must be two positive integers $U$ and $V$ such that

$$
e(x ; a)=0 \text { if } a>V \text { or } a<-U,
$$

while

$$
e(x ; V)>0 \text { and } e(x ;-U)>0
$$

and two relatively prime positive integers $a$ and $b$ such that

$$
e(x ; a)>0 \text { and } e(x,-b)>0 .
$$

Conditions (2.1) and (2.2) are imposed to allow a matrix formulation for the investigation of the problem, and are not probabilistic in nature, whereas condition (2.3) is to insure that the RWPE is irreducible.

Recurrence and transience criteria are given by examining the eigenvalues of the $(V+U) \times(V+U)$ matrix $Q$ defined by

$$
Q=A_{1} A_{2} \cdots A_{N}
$$

where $A_{x}$ is the $(V+U) \times(V+U)$ matrix given by

$$
\begin{aligned}
A_{x}(i, k) & =\delta(i+1, k) \text { if } 1 \leq i<V+U, \\
A_{x}(V+U, k) & =\frac{\delta(V+1, k)-e(x ; V+1-k)}{e(x ;-U)} .
\end{aligned}
$$

If we let $d_{1} \leq d_{2} \leq \cdots \leq d_{V+U}$ denote the logarithms of the moduli of the eigenvalues of $Q$, repeated according to algebraic multiplicities, it is shown that either $d_{V}=0$ or $d_{V+1}=0$, and

TheOrem 2.2 ([5], Theorem 35). Let $X$ be a RWPE with period $N$ and suppose that (2.1), (2.2) and (2.3) are satisfied.

- If $d_{V}+d_{V+1}>0$ then $\operatorname{Pr}\left(\lim _{t \rightarrow \infty} X_{t}=+\infty\right)=1$.

- If $d_{V}+d_{V+1}=0$ then $\operatorname{Pr}\left(-\infty=\liminf _{t \rightarrow \infty} X_{t}<\lim \sup _{t \rightarrow \infty} X_{t}=+\infty\right)=1$. ( $X$ is recurrent).

- If $d_{V}+d_{V+1}<0$ then $\operatorname{Pr}\left(\lim _{t \rightarrow \infty} X_{t}=-\infty\right)=1$. 
To apply this theorem we need to determine the moduli of at least $V+1$ of the eigenvalues of $Q$, which may not be possible. However, it is clear from the proof of this theorem that 1 is an eigenvalue of $Q$, and that if the geometric multiplicity of 1 is 1 while the algebraic multiplicity of 1 is greater than 1 , then $d_{V}+d_{V+1}=0$ and the RWPE is recurrent.

What is more, observe that if $q(t)$ denotes the characteristic polynomial of $Q$, then in the case of an ordinary random walk, where $N=1$,

$$
q^{\prime}(1)=-e(x ;-U) \mu
$$

Thus, in the case of a random walk $X$ satisfying (2.1), (2.2) and (2.3), $q^{\prime}(1)=0$ if and only if $X$ is recurrent.

We will extend this last result to the case where $N>1$ in the following way. We will show that 1 is the only eigenvalue of $Q$ of modulus 1 , so if $q^{\prime}(1) \neq 0$, then $d_{V}+d_{V+1} \neq 0$, and the RWPE is not recurrent. We will also prove that the geometric multiplicity of 1 is always 1 so if $q^{\prime}(1)=0$, then the algebraic multiplicity of 1 is greater than $1, d_{V}+d_{V+1}=0$ and the RWPE is recurrent.

It is easy to show that if $A_{x}$ is the matrix defined above, then

$$
\begin{aligned}
& A_{x}^{-1}(1, k)=\frac{\delta(V, k)-e(x ; V-k)}{e(x ; V)} \\
& A_{x}^{-1}(i, k)=\delta(i, k+1) \text { if } i \geq 2
\end{aligned}
$$

so each $A_{x}^{-1}$ is a companion matrix. Since an eigenvalue $\lambda$ of an invertible matrix $M$ is geometrically simple iff $1 / \lambda$ is a geometrically simple eigenvalue of $M^{-1}$, we can determine the geometric simplicity of 1 as an eigenvalue of $Q$ by examining whether or not 1 is a geometrically simple eigenvalue of a product of companion matrices. We will show that under certain conditions, if $\lambda>0$ is a common eigenvalue of a finite set of companion matrices, then $\lambda$ is a geometrically simple eigenvalue of their product. The matrices arising from RWPE's satisfying conditions (2.1), (2.2) and (2.3) will satisfy these conditions.

3. A Formula for the Product of Companion Matrices. We consider $n$ companion matrices $B_{1}, \ldots, B_{n}$ of dimension $n$. The first row of $B_{j}$ is denoted by

$$
\left[\begin{array}{llllll}
a_{j, 1} & a_{j, 2} & a_{j, 3} & \ldots & a_{j, n-1} & a_{j, n}
\end{array}\right]
$$

For $k=1, \ldots, n$ we define the upper triangular matrix

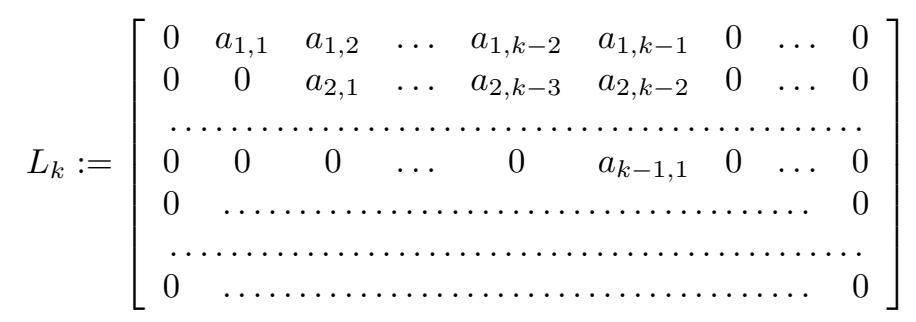


and the matrix

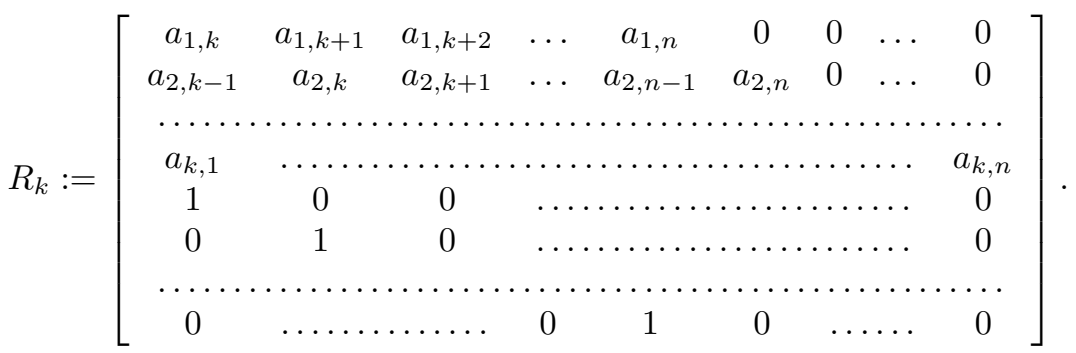

Note that each entry of the first row of $B_{j}, j=1, \ldots, k$, appears in either the $j$ th row of $L_{k}$ or $R_{k}$. For example, if $n=4, k=3$, then

$$
L_{3}=\left[\begin{array}{cccc}
0 & a_{1,1} & a_{1,2} & 0 \\
0 & 0 & a_{2,1} & 0 \\
0 & 0 & 0 & 0 \\
0 & 0 & 0 & 0
\end{array}\right] \quad R_{3}=\left[\begin{array}{cccc}
a_{1,3} & a_{1,4} & 0 & 0 \\
a_{2,2} & a_{2,3} & a_{2,4} & 0 \\
a_{3,1} & a_{3,2} & a_{3,3} & a_{3,4} \\
1 & 0 & 0 & 0
\end{array}\right]
$$

and, if $n=k=4$, then

$$
L_{4}=\left[\begin{array}{cccc}
0 & a_{1,1} & a_{1,2} & a_{1,3} \\
0 & 0 & a_{2,1} & a_{2,2} \\
0 & 0 & 0 & a_{3,1} \\
0 & 0 & 0 & 0
\end{array}\right] \quad R_{4}=\left[\begin{array}{cccc}
a_{1,4} & 0 & 0 & 0 \\
a_{2,3} & a_{2,4} & 0 & 0 \\
a_{3,2} & a_{3,3} & a_{3,4} & 0 \\
a_{4,1} & a_{4,2} & a_{4,3} & a_{4,4}
\end{array}\right]
$$

If we define $\operatorname{sgn}(x):=-1,0,1$ if $x<0,=0,>0$, respectively, and $a_{i, 0}:=a_{i, n}$, then for any positive real number $r$,

$$
\operatorname{sgn}\left(\left(R_{n}+r L_{n}\right)_{i, j}\right)=\operatorname{sgn}\left(a_{i,(j-i) \bmod n}\right) .
$$

Let $I$ denote the $n \times n$ identity matrix.

LEMMA 3.1. For $k=1, \ldots, n$, we have

$$
B_{1} B_{2} \ldots B_{k}=\left(I-L_{k}\right)^{-1} R_{k}
$$

Proof. We use induction on $k$. The equation is true for $k=1$ because $L_{1}=0$ and $R_{1}=B_{1}$. For the induction step from $k-1$ to $k$ we have to verify the equation

$$
\left(I-L_{k}\right)\left(I-L_{k-1}\right)^{-1} R_{k-1} B_{k}=R_{k} .
$$

Now $Q:=\left(I-L_{k}\right)\left(I-L_{k-1}\right)^{-1}$ is the identity matrix with the $k$ th column replaced by

$$
\left[\begin{array}{llllllll}
-a_{1, k-1} & -a_{2, k-2} & \ldots & -a_{k-1,1} & 1 & 0 & \ldots & 0
\end{array}\right]^{t} .
$$

The product $Q R_{k-1}$ is the same as $R_{k-1}$ except that the entries in the first column which lie in the first $k-1$ rows are replaced by 0 . Now the desired equation $Q R_{k-1} B_{k}=R_{k}$ follows easily. 
Theorem 3.2. Let $1 \leq k \leq n$ and $\lambda \in \mathbb{C}$. Put $M:=B_{1} B_{2} \cdots B_{k}, L:=L_{k}$, $R:=R_{k}$. Then $\lambda$ is an eigenvalue of $M$ iff $\lambda$ is an eigenvalue of $R+\lambda L$; in the case that $\lambda$ is an eigenvalue of both $M$ and $R+\lambda L$, the $\lambda$-eigenspaces for both matrices are the same. Moreover,

$$
\operatorname{det}(M-\mu I)=\operatorname{det}(R+\mu L-\mu I) .
$$

Proof. Lemma 3.1 implies that

$$
M-\lambda I=(I-L)^{-1}(R-\lambda(I-L)) .
$$

This equation shows that $\operatorname{ker}(M-\lambda I)=\operatorname{ker}(R+\lambda L-\lambda I)$. We complete the proof by noting that $\operatorname{det}(M-\mu I)=\operatorname{det}(I-L)^{-1} \operatorname{det}(R+\mu L-\mu I)$ and $\operatorname{det}(I-L)=1$.

For example, if $n=k=4$,

$$
R_{4}+\mu L_{4}-\mu I=\left[\begin{array}{cccc}
a_{1,4}-\mu & \mu a_{1,1} & \mu a_{1,2} & \mu a_{1,3} \\
a_{2,3} & a_{2,4}-\mu & \mu a_{2,1} & \mu a_{2,2} \\
a_{3,2} & a_{3,3} & a_{3,4}-\mu & \mu a_{3,1} \\
a_{4,1} & a_{4,2} & a_{4,3} & a_{4,4}-\mu
\end{array}\right]
$$

By (3.5), the determinant of this matrix is equal to the characteristic polynomial of $B_{1} B_{2} B_{3} B_{4}$.

4. The Multiplicity of Common Eigenvalues. Our criterion for geometrically simple positive eigenvalues will be stated in terms of the irreducibility of certain nonnegative matrices. Recall that an $n \times n$ matrix $E$ is said to be irreducible if the index set $\{1,2, \ldots, n\}$ cannot be split into two proper complementary sets (without common indices $)\left\{i_{1}, \ldots, i_{\mu}\right\}$ and $\left\{j_{1}, \ldots, j_{\nu}\right\},(\nu>0, \mu>0, \mu+\nu=n)$ such that

$$
E_{i_{\alpha}, j_{\beta}}=0 \quad(\alpha \in\{1, \ldots, \mu\}, \beta \in\{1, \ldots, \nu\}) .
$$

The matrix $E$ is called nonnegative if all of its entries are nonnegative.

THEOREM 4.1. For $k \leq n$, let $B_{1}, B_{2}, \ldots, B_{k}$ be $n \times n$ companion matrices with a common positive eigenvalue $x$. Form $R:=R_{k}$ and $L:=L_{k}$ according to (3.2) and (3.3). If there are matrices $B$ and $C$, with $C$ being invertible, such that $R+x^{k} L=C^{-1}-B$, and $C\left(x^{k} I+B\right)$ is nonnegative and irreducible, then $x^{k}$ is a geometrically simple eigenvalue of $M:=B_{1} B_{2} \cdots B_{k}$.

Proof. It follows from Theorem 3.2 that $\chi:=\left[x^{n-1}, x^{n-2}, \ldots, x, 1\right]^{t}$ is an eigenvector of $R+x^{k} L$ with all positive entries. Since $R+x^{k} L=C^{-1}-B$, we know that $C\left(x^{k} I+B\right) \chi=\chi$. We have assumed that $C\left(x^{k} I+B\right)$ is an irreducible nonnegative matrix. It then follows from Remark 3 following Frobenius' theorem in [3, pp. 50-64] that $\chi$ is the unique eigenvector (up to constant factors) of the nonnegative matrix $C\left(x^{k} I+B\right)$. It then follows from Frobenius' theorem itself that 1 is the eigenvalue of $C\left(x^{k} I+B\right)$ of maximum modulus, and that 1 is an algebraically simple eigenvalue of $C\left(x^{k} I+B\right)$. Therefore 1 is a geometrically simple eigenvalue of $C\left(x^{k} I+B\right)$.

Since $\left(R+x^{k} L\right) \nu=x^{k} \nu$ iff $C\left(x^{k} I+B\right) \nu=\nu, x^{k}$ is a geometrically simple eigenvalue of $R+x^{k} L$. 
It then follows from Theorem 3.2 that $x^{k}$ is a geometrically simple eigenvalue of $M$.

As an example, consider the case where $n=k=3$. Assume $x>0$ is a common eigenvalue for $B_{1}, B_{2}, B_{3}$. Then there are constants $a_{i}$ and $b_{i}$ so that

$$
B_{i}=\left[\begin{array}{ccc}
x-a_{i} & a_{i} x-b_{i} & x b_{i} \\
1 & 0 & 0 \\
0 & 1 & 0
\end{array}\right]
$$

for $i=1,2,3$. We have

$$
R+x^{3} L=\left[\begin{array}{ccc}
x b_{1} & x^{3}\left(x-a_{1}\right) & x^{3}\left(a_{1} x-b_{1}\right) \\
x a_{2}-b_{2} & x b_{2} & x^{3}\left(x-a_{2}\right) \\
x-a_{3} & x a_{3}-b_{3} & x b_{3}
\end{array}\right]
$$

If we assume that for each $i$ we have $\left(x^{2}-b_{i}\right) /\left(x-a_{i}\right)>0$ and $\left(b_{i}-x a_{i}\right) /\left(x-a_{i}\right)>0$ and we write

$$
\begin{aligned}
C^{-1} & =\left[\begin{array}{ccc}
0 & x^{3}\left(x-a_{1}\right) & 0 \\
0 & 0 & x^{3}\left(x-a_{2}\right) \\
x-a_{3} & 0 & 0
\end{array}\right] \\
B & =\left[\begin{array}{ccc}
-x b_{1} & 0 & x^{3}\left(b_{1}-x a_{1}\right) \\
b_{2}-x a_{2} & -x b_{2} & 0 \\
0 & b_{3}-x a_{3} & -x b_{3}
\end{array}\right],
\end{aligned}
$$

then $R+x^{3} L=C^{-1}-B$ and $C\left(x^{3} I+B\right)$ is an irreducible matrix with no negative entries.

In what follows we will apply Theorem 4.1 directly only if $k=n$, in which case the matrix $R+x^{k} L$ is particularly simple. When the number of factors $k$ is different from the dimension $n$ of the factors we can reduce the case $k=n$ as follows. The proof of Corollary 4.3 below is an example of this technique.

Case 1: $n<k$ In this case, to each $B_{j}$ we associate a $k \times k$ companion matrix $B_{j}^{\prime}$ whose first row is given by $\left(B_{j}^{\prime}\right)_{1, y}=\left(B_{1}\right)_{1, y}$ if $y \in\{1, \ldots, n\}$ and $\left(B_{j}^{\prime}\right)_{1, y}=0$ if $y \in\{n+1, \ldots, k\}$. We can then apply Theorem 4.1 to $M^{\prime}:=B_{1}^{\prime} \cdots B_{k}^{\prime}$, and give a sufficient condition for $x^{k}$ to be a geometrically simple eigenvalue of $M^{\prime}$. It remains to show that $x^{k}$ is a geometrically simple eigenvalue of $M$ if $x^{k}$ is a geometrically simple eigenvalue of $M^{\prime}$. Note that $x^{k}$ is an eigenvalue of both $M$ and $M^{\prime}$, and one eigenvector associated with $x^{k}$ is $\chi$ (with the appropriate dimension).

To finish the proof, observe that each $B_{j}^{\prime}$ is lower block triangular, where the "northwest block" is the $n \times n$ matrix $B_{j}$, the "northeast block" is the $n \times(k-n)$ zero matrix, and the "southeast block" is an $(k-n) \times(k-n)$ nilpotent matrix. Since $k>k-n$, the last $k-n$ columns of $M^{\prime}$ are all zero, and the "northwest block" of $M^{\prime}$ is $M$. Let $S$ denote the $(k-n) \times n$ "southwest block" of $M^{\prime}$.

Suppose that $x^{k}$ is a geometrically simple eigenvalue of $M^{\prime}$. If $M \nu=x^{k} \nu$, form the $k$ dimensional column vector $\nu^{\prime}$ whose first $n$ coordinates are $\nu$ 
and whose last $k-n$ coordinates are $x^{-k} S \nu$. Then $M^{\prime} \nu^{\prime}=x^{k} \nu^{\prime}$. Therefore, $\left(\nu^{\prime}\right)_{j}=x^{k-j}$ for $j \in\{1, \ldots, k\}$. Therefore $(\nu)_{j}=c x^{k-n} x^{n-j}$ for $j \in\{1,2, \ldots, n\}$ for some constant $c$, so the eigenspace of $x^{k}$ for $M$ is one dimensional.

Case 2: $n>k$ This case quickly reduces to Case 1 or to Theorem 4.1. First observe that for any matrix $A, a$ is a geometrically simple eigenvalue of $A$ if $a^{s}$ is a geometrically simple eigenvalue of $A^{s}$. (The converse is not true unless the sth powers of the eigenvalues of $A$ are distinct. This becomes apparent by considering the Jordan normal form of $A$.) Hence it will be sufficient to show that $x^{s k}$ is a geometrically simple eigenvalue of $M^{s}$ for some $s$.

Let $s$ be any integer so that $s k \geq n$. If $s k=n$ then apply Theorem 4.1 to the product $M^{s}$. If $s k>n$, proceed as in Case 1 .

The first row of the companion matrices arising from the study of RWPE's have additional structure which makes it easy to see one way to apply Theorem 4.1:

- There is always exactly one positive entry, and it always appears in the $V^{\text {th }}$ column.

Bearing this in mind, we say that a companion matrix is Type $p$ if there is a positive integer $p$ such that

- The matrix has a positive entry in the first row and $p^{\text {th }}$ column;

- All other entries in the first row are nonpositive.

It is clear that the techniques described above in Case 1 will preserve the Type $p$ property of a set of companion matrices.

Suppose that $B_{1}, B_{2}, \ldots, B_{n}$ are $n \times n$ type $p$ companion matrices, $r$ is any given positive number, and $L_{n}$ and $R_{n}$ are defined as in (3.2) and (3.3), respectively. Let $D:=R_{n}+r L_{n}$. In the following discussion, let

$$
[j]:=\left\{\begin{aligned}
n & \text { if } j \text { is divisible by } n \\
j \bmod n & \text { otherwise. }
\end{aligned}\right.
$$

According to (3.4), $D_{i, j}>0$ iff $a_{i,[j-i]}>0$ iff $[j-i]=p$. Therefore, if we define the nonnegative matrix $C$ by

$$
C_{j, i}:=\left\{\begin{aligned}
0 & \text { if } D_{i, j} \leq 0 \\
1 / D_{i, j} & \text { if } D_{i, j}>0
\end{aligned}\right.
$$

we see that $C_{j, i}>0$ iff $j=[p+i]$. Therefore, the matrix $F$ defined by $F_{i, j}=\operatorname{sgn}\left(C_{i, j}\right)$ is a cyclic permutation matrix, from which we see that $C$ itself is invertible with

$$
C_{i, j}^{-1}=\left\{\begin{aligned}
0 & \text { if } D_{i, j} \leq 0, \\
D_{i, j} & \text { if } D_{i, j}>0 .
\end{aligned}\right.
$$

Furthermore, it is clear from (4.2) that if we put $B=C^{-1}-D$, then $B$ is a nonnegative matrix as well. Hence $C B$ is nonnegative and $R_{n}+r L_{n}=C^{-1}-B$.

Thus, if we want to apply Theorem 4.1 to products of Type p companion matrices, we can give conditions under which $C\left(x^{n} I+B\right)$ is irreducible for this particular decomposition. This means we need to identify the locations of the positive entries of $C B$ as well as those of the positive entries of $C$. 
To see where the positive entries of $C B$ are, note that

$$
(C B)_{i, j}=C_{i,[i-p]} B_{[i-p], j}
$$

so $(C B)_{i, j}>0$ iff $B_{[i-p], j}>0$. From the definitions of $B$ and $C$ and from (4.2) we see that $(C B)_{i, i}=0$, and that for $i \neq j,(C B)_{i, j}>0$ iff $a_{[i-p],[j-i+p]}<0$. This can be summarized as

$$
(C B)_{i,[i+d]}>0 \text { iff } a_{[i-p],[d+p]}<0
$$

for any integer $d$ and any integer $i \in\{1,2, \ldots, n\}$.

TheOREM 4.2. Suppose that $n>1$ and $f x p \in\{1,2, \ldots, n\}$. Let $B_{1}, B_{2}, \ldots, B_{n}$ be $n \times n$ Type $p$ companion matrices. Let $x>0$ be given, and define $C$ and $B$ as in the preceding discussion. Then $C\left(x^{n} I+B\right)$ is irreducible under any of the following conditions.

(i) $p$ and $n$ are relatively prime.

(ii) There exists $q \in\{1,2, \ldots, n\}$ such that each of the matrices $B_{j}$ has a strictly negative entry in its first row and $q^{\text {th }}$ column, and $|p-q|$ and $n$ are relatively prime.

(iii) There exist $c, d \in\{1,2, \ldots, n\}$ with $c<p<n$ such that each of the matrices $B_{j}$ has a strictly negative entry in its first row and $c^{\text {th }}$ and $d^{\text {th }}$ columns, and $p-c$ and $d-p$ are relatively prime.

Proof. If $A$ is any matrix, let $\operatorname{sgn}(A)$ be the matrix defined by $\operatorname{sgn}(A)_{i, j}=$ $\operatorname{sgn}\left(A_{i, j}\right)$.

Note that $E:=C\left(x^{n} I+B\right)$ is irreducible if any of the following matrices is irreducible: $\operatorname{sgn}(E), \operatorname{sgn}(C), \operatorname{sgn}(C B)$.

Suppose that (i) holds. Consider the directed graph on the cyclic group $\mathbb{Z}_{n}$ whose incidence matrix is $\operatorname{sgn}(C)$. From the discussion following (4.1), for each node $g$ there is an edge on this graph from $g$ to $g-p$. Therefore, for any integer $m$ and any node $g$ there is a path from $g$ to $g-m p$. Since $n$ and $p$ are relatively prime there is a path from $g$ to $g-1$, so all the nodes in the graph are connected, and $\operatorname{sgn}(C)$ is irreducible.

Suppose that (ii) holds. Consider the directed graph on $\mathbb{Z}_{n}$ whose incidence matrix is $\operatorname{sgn}(C B)$. It follows from (4.3) that for each node $g$ there is an edge from $g$ to $g+(p-q)$. Since $|p-q|$ and $n$ are relatively prime, it follows (by the same argument as for (i)) that $\operatorname{sgn}(C B)$ is irreducible.

Suppose that (iii) holds. Consider the directed graph on $\mathbb{Z}_{n}$ whose incidence matrix is $\operatorname{sgn}(C B)$. It follows from (4.3) that for each node $g$ there is an edge from $g$ to $g+c-p$ and from $g$ to $g+d-p$. Since $p-c$ and $d-p$ are relatively prime, it follows (by the same argument as for (i)) that $\operatorname{sgn}(C B)$ is irreducible.

Corollary 4.3. Suppose that $n>1$ and fix $p \in\{1,2, \ldots, n\}$. Let $B_{1}, B_{2}, \ldots, B_{k}$ be $k n \times n$ Type $p$ companion matrices with common eigenvalue $x>0$. Then $x^{k}$ is a geometrically simple eigenvalue of the product $B_{1} B_{2} \cdots B_{k}$ under any of the following additional conditions.

(i) $p$ and $k$ are relatively prime.

(ii) There exists $q \in\{1,2, \ldots, n\}$ such that each of the matrices $B_{j}$ has a strictly negative entry in its first row and $q^{\text {th }}$ column, and $|p-q|$ and $n$ are relatively prime. 
(iii) There exist $c, d \in\{1,2, \ldots, n\}$ with $c<p<d$ such that each of the matrices $B_{j}$ has a strictly negative entry in its first row and $c^{\text {th }}$ and $d^{\text {th }}$ columns, and $p-c$ and $d-p$ are relatively prime.

Proof. We assume first that $k=n$. We apply Theorem 4.1 and Theorem 4.2.

If $k>n$ we use the method of Case 1 discussed above to reduce to the case just treated.

If $k=1$ the theorem is obviously true.

If $1<k<n$ we use the method of Case 2 with $s$ a power of $k$.

As an example, consider

$$
\begin{array}{cc}
B_{1}=\left[\begin{array}{cccc}
-\frac{253}{26} & 20 & -\frac{215}{26} & -1 \\
1 & 0 & 0 & 0 \\
0 & 1 & 0 & 0 \\
0 & 0 & 1 & 0
\end{array}\right] \quad B_{2}=\left[\begin{array}{cccc}
-\frac{1}{5} & \frac{12}{5} & -\frac{2}{5} & -\frac{4}{5} \\
1 & 0 & 0 & 0 \\
0 & 1 & 0 & 0 \\
0 & 0 & 1 & 0
\end{array}\right] \\
B_{3}=\left[\begin{array}{cccc}
-1 & 4 & -1 & -1 \\
1 & 0 & 0 & 0 \\
0 & 1 & 0 & 0 \\
0 & 0 & 1 & 0
\end{array}\right] B_{4}=\left[\begin{array}{cccc}
-1 & \frac{9}{2} & -\frac{3}{2} & -1 \\
1 & 0 & 0 & 0 \\
0 & 1 & 0 & 0 \\
0 & 0 & 1 & 0
\end{array}\right] .
\end{array}
$$

It is easy to check that these matrices have the common eigenvalue 1 and the common eigenvector $[1,1,1,1]^{t}$. We have

$$
R+1^{4} L=\left[\begin{array}{cccc}
0 & 0 & 20 & 0 \\
0 & 0 & 0 & \frac{12}{5} \\
4 & 0 & 0 & 0 \\
0 & \frac{9}{2} & 0 & 0
\end{array}\right]-\left[\begin{array}{cccc}
1 & \frac{253}{26} & 0 & \frac{215}{26} \\
\frac{2}{5} & \frac{4}{5} & \frac{1}{5} & 0 \\
0 & 1 & 1 & 1 \\
1 & 0 & \frac{3}{2} & 1
\end{array}\right]
$$

from which it is clear that the hypotheses of Corollary 4.3 are satisfied.

Notice that it is not sufficient simply to assume that the matrices are Type $p$. For example, consider $k=n=2, p=2, r=1$ and

$$
B_{1}=B_{2}=\left[\begin{array}{ll}
0 & 1 \\
1 & 0
\end{array}\right] \text {. }
$$

Then 1 is an eigenvalue of $B_{1} B_{2}=I$ with geometric multiplicity 2 .

The following example shows that not having the Type $p$ property can also lead to an eigenvalue with geometric multiplicity larger than 1. Put

$$
B_{k}=\left[\begin{array}{ccc}
b_{k} & 1 & -b_{k} \\
1 & 0 & 0 \\
0 & 1 & 0
\end{array}\right]
$$

for $k=1,2$. Then both $[1,1,1]^{t}$ and $[0,1,0]^{t}$ are eigenvectors for $B_{1} B_{2}$ with the eigenvalue 1 . 
5. Application to Random Walks in Periodic Environments. In this section we use the results of the previous sections to prove the following theorem.

THEOREM 5.1. Suppose that $X$ is a random walk in a periodic environment with period $N$ satisfying (2.1), (2.2) and (2.3). Let $Q$ be the matrix defined in (2.4), and let $q$ be the characteristic polynomial of $Q$. Then $X$ is recurrent iff $q^{\prime}(1)=0$.

Proof. As noted in Section 2, in order to prove that $q^{\prime}(1)=0$ implies that $X$ is recurrent we need only prove that 1 is a geometrically simple eigenvalue of $Q$. To do so we observe that we can apply Corollary 4.3 to $Q^{-1}$. The integer $p$ in Corollary 4.3 is $V, p-c=a$ and $d-p=b$, where $a$ and $b$ are as defined in (2.3). In either case we conclude that 1 is a geometrically simple eigenvalue of $Q^{-1}$, and, therefore, 1 is a geometrically simple eigenvalue of $Q$.

To prove the converse it is sufficient to show that 1 is the only root of $q$ of modulus 1. To do so, let $P$ denote the transition matrix of $X$. It follows from (2.3) that $X$ is irreducible. Observe that if $P f=f$ and that for some state $s,|f(s)| \geq|f(t)|$ for all states $t$, then $f(t)=f(s)$ for all states $t$.

Suppose now that $g \in \mathbb{C}^{\mathbb{Z}}$. For each $x \in \mathbb{Z}$ define $g^{x} \in \mathbb{C}^{U+V}$ by $g^{x}(j)=$ $g(x+V+1-j)$. It is easy to check that the following are equivalent.

- $P g=g$.

- $A_{x} g^{x}=g^{x-1}$ for all $x \in \mathbb{Z}$, where $A_{x}$ is defined following (2.4).

- $g^{x}=B_{x} g^{x-1}$ for all $x \in \mathbb{Z}$, where $B_{x}=A_{x}^{-1}$.

Finally observe that given any $v \in \mathbb{C}^{U+V}$ if we define $f \in \mathbb{C}^{\mathbb{Z}}$ by

- $f(x)=v(V+1-x)$ for $x \in\{-U+1,-U+2, \ldots, V\}$,

- $f(x)=$ the first entry in $B_{x-V} \cdots B_{1} v$ for $x>V$,

- $f(x)=$ the last entry in $A_{x+U} \cdots A_{0} v$ for $x \leq-U$

and use the aforementioned equivalences then $P f=f$. Therefore, if we choose $v$ to be an eigenvector of $Q^{-1}$ whose eigenvalue has unit modulus then we have some $f \in \mathbb{C}^{\mathbb{Z}}$ such that $P f=f$ and $|f(x)|$ is a periodic function of $x$. Therefore $f$ has an entry of maximum modulus, so $f$ is constant. This tells us that $v$ is constant. Since constant vectors are eigenvectors of $Q^{-1}$ for the eigenvalue 1 , the only eigenvalue of $Q^{-1}$, and of $Q$, of unit modulus is the eigenvalue 1 .

As an example, consider the case $U=V=2, k=4$ and the transition probabilities $e(a, b)$ given by

$$
\left[\begin{array}{lllll}
e(0,-2) & e(0,-1) & e(0,0) & e(0,1) & e(0,2) \\
e(1,-2) & e(1,-1) & e(1,0) & e(1,1) & e(1,2) \\
e(2,-2) & e(2,-1) & e(2,0) & e(2,1) & e(2,2) \\
e(3,-2) & e(3,-1) & e(3,0) & e(3,1) & e(3,2)
\end{array}\right]=\left[\begin{array}{ccccc}
\frac{1}{5} & \frac{3}{10} & \frac{1}{10} & \frac{1}{5} & \frac{1}{5} \\
\frac{1}{20} & \frac{43}{104} & 0 & \frac{253}{520} & \frac{1}{20} \\
\frac{1}{3} & \frac{1}{6} & 0 & \frac{1}{12} & \frac{5}{12} \\
\frac{1}{4} & \frac{1}{4} & 0 & \frac{1}{4} & \frac{1}{4}
\end{array}\right]
$$

The matrices $B_{1}, B_{2}, B_{3}, B_{4}$ are those in the example following the proof of Corollary 4.3 .

We see from Corollary 4.3 (without calculations) that 1 is a geometrically simple eigenvalue of the corresponding matrix $M=Q^{-1}$, and therefore of $Q$. The matrix 
$R_{4}+\mu L_{4}-\mu I$ in Theorem 4.1 is

$$
\left[\begin{array}{cccc}
-1-\mu & -\frac{253}{26} \mu & 20 \mu & -\frac{215}{26} \mu \\
-\frac{2}{5} & -\frac{4}{5}-\mu & -\frac{1}{5} \mu & \frac{12}{5} \mu \\
4 & -1 & -1-\mu & -\mu \\
-1 & \frac{9}{2} & -\frac{3}{2} & -1-\mu
\end{array}\right]
$$

It follows that the characteristic polynomial of $M$ is

$$
\mu^{4}-\frac{2013}{13} \mu^{3}+\frac{19987}{65} u^{2}-\frac{10039}{65} \mu+\frac{4}{5}=\frac{1}{65}(\mu-1)^{2}\left(65 \mu^{2}-9935 \mu+52\right),
$$

so the RWPE is recurrent.

\section{REFERENCES}

[1] B. Derrida. Velocity and diffusion constant of a periodic one-dimensional hopping model. Journal of Statistical Physics, 31:433-450, 1983.

[2] T. Fort. Finite Differences and Difference Equations in the Real Domain. Oxford at the Clarendon Press, London, 1948.

[3] F. R. Gantmacher. The Theory of Matrices, Volume 2. Chelsea Publishing Company, New York, NY, 1959.

[4] D. J. Hartfiel. Nonhomogeneous Matrix Products. World Scientific, Singapore, 2002.

[5] E. S. Key. Recurrence and transience criteria for random walk in a random environment. The Annals of Probability, 12:529-560, 1984.

[6] V. Lakshmikantham and D. Trigiante. Theory of Difference Equations: Numerical Methods and Applications. Academic Press, Boston, 1988.

[7] J. Popenda. Finite difference equations and periodicity, in New Developments in Difference Equations and Applications, Proceedings of the Third International Conference on Difference Equations. S. S. Cheng, S. Elaydi and G. Ladas, eds, Gordon and Breach, Amsterdam, 1999. 